\title{
Characterization of Neutral Loop Discharges in the VINETA Device
}

\author{
Adrian von Stechow ${ }^{1}$, Olaf Grulke ${ }^{1}$, and Thomas Klinger ${ }^{1}$ \\ Max-Planck-Institut für Plasmaphysik, Greifswald
}

July 23,2012

\begin{abstract}
A neutral loop (NL) discharge has been implemented in the linear plasma device VINETA with three different antenna configurations. Radial profile measurements of the electron density, temperature and floating potential are performed at various neutral loop radii and compared to single particle simulations. Local density maxima are observed near the NL, for which several mechanisms have been proposed. The measured temperature profiles do not support the model of a thermal heating process, whereas the floating potential profiles corroborate the assumption of a locally enhanced induction current.
\end{abstract}

\section{Introduction}

A neutral loop (NL) is defined as a closed line along which the absolute magnetic field vanishes. Such a configuration can easily be created by arranging at least three magnetic field coils with opposing currents around a cylindrical vacuum vessel, thus creating an azimuthal X-line $[1,2]$. If a radio frequency ( $\mathrm{rf}$ ) antenna is operated in the vicinity of the NL, a so-called neutral loop discharge (NLD) is started. Previous experiments have provided evidence of an increased plasma production near the NL, which is characterized by an increased local optical emission and plasma density which correlates with the NL radius [3]. Due to the well controlled density profile, these discharges can be used for precise plasma etching via the direct control of the NL radius [4]. Several concurrent plasma production mechanisms have been proposed to account for the increased plasma density near the NL $[1,5-9]$.

The most basic model suggests that the plasma current induced by the loop antenna is enhanced near the magnetic null point [1]. More advanced models assume a collisionless heating process [5], which has also been investigated in numerical simulations [6]: In this model, the single particle motion of electrons in a magnetic neutral point configuration with a time-varying electrical field leads to chaotic meandering particle trajectories, thereby raising anomalous resistivity and increasing the local electron temperature [7] and plasma density. Recently, local resonant heating in regions where the electron cyclotron frequency corresponds to the rf frequency has been discussed [8]. This process is called partial electron cyclotron resonance (PECR). Finally, a collisionless wave damping mechanism in the complex magnetic field of the NL setup has been proposed [9]. In that setup, a helicon wave field is observed that is modified by the presence of a magnetic NL configuration. The temperature increase is interpreted as local kinetic wave damping.

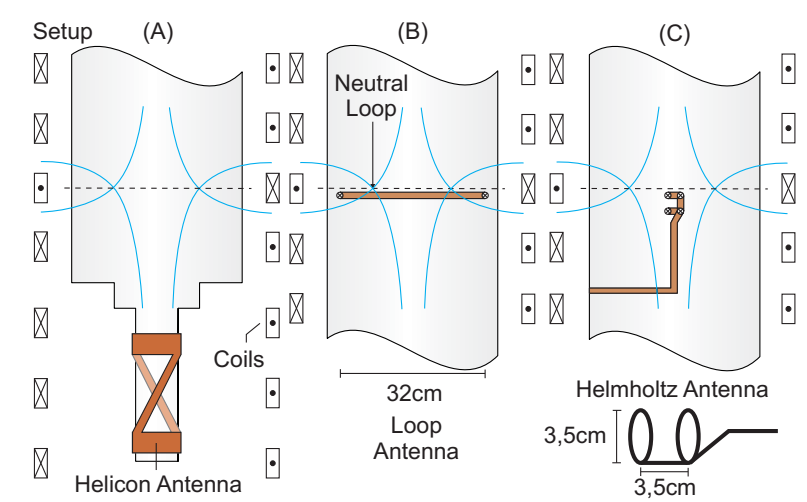

Fig. 2. Diagram of the $3 \mathrm{rf}$ antenna configurations in the plasma vessel, including the magnetic field coils and the resulting separatrices (blue).

Summarizing these findings, the processes responsible for the observations (increased plasma density, electron temperature and optical emission) remains under discussion. In the present paper, NLDs created by different antenna geometries are investigated in order to clarify the role of the neutral loop position relative to that of the $\mathrm{rf}$ antenna and its electric field.

\section{Experimental Setup}

The linear plasma device VINETA [10], shown schematically in Fig. 1, consists of 4 cylindrical modules surrounded by 36 magnetic field coils. Together with 5 independent power supplies, this setup allows for a versatile configuration of the magnetic field. In the present paper, a NL was created similar to previous experiments by superimposing the homogeneous field, created by 10 coils of equal polarity and current (green in fig. 1), and the field of a single coil 


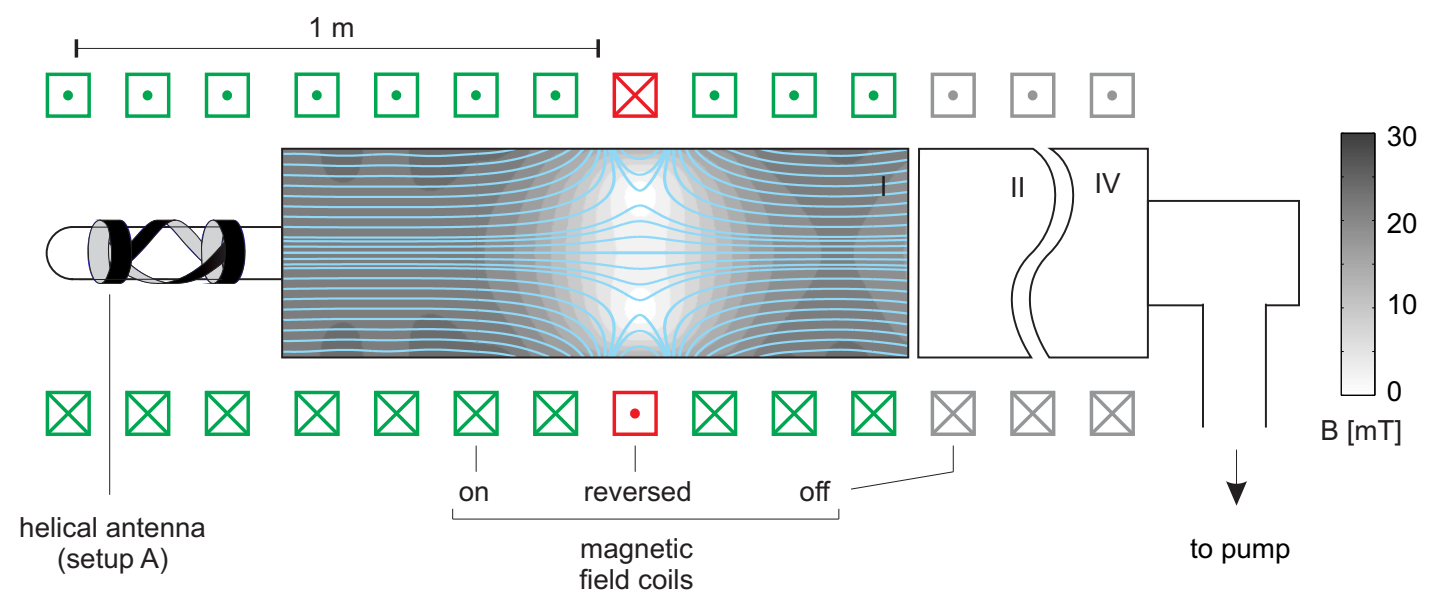

Fig. 1. Diagram of the VINETA experiment. Additionally, the total magnetic field (color coded) and its field lines (blue) are shown.

(red in Fig. 1) with reversed polarity and a significantly higher current. The calculated resulting magnetic field in the radial-axial plane for a specific ratio of coil currents is shown in fig. 1 together with the resulting field lines. Due to the high sensitivity of the NL position to variations of the coil currents and positions, the magnetic field was measured with a three-axis hall probe for each configuration. The measurements agree well with the calculation (not shown).

For plasma production, the three radio frequency (rf) antenna configurations shown in fig. 2 were used. The aim of setup A is to create a plasma at a distance far from the NL (further than the collisionless skin depth $\delta_{\text {skin }}$ ). Due to the negligible influence of the rf field at the NL, the plasma is expected to essentially follow the field lines into the NL region and to exhibit complex trajectories and particle trapping in the static magnetic field. For this configuration, a helical antenna [11] is operated in a capacitive discharge mode at $30 \mathrm{~W}$ input power, which results in a density maximum near the antenna radius at $r=5 \mathrm{~cm}$. The magnetic field is configured such that the separatrices magnetically connect the NL to this region. In setup $\mathrm{B}$, a single loop antenna with a radius of $16 \mathrm{~cm}$ is located inside the vacuum vessel, axially near the NL. At $60 \mathrm{~W}$ rf power, typical plasma densities of $n \approx 10^{15} \mathrm{~m}^{-3}$ are produced which corresponds to a collisionless skin depth of $\delta_{\text {skin }} \approx 0.17 \mathrm{~m}$. Thus, the neutral loop is always within a skin depth of the rf antenna. Finally, in order to reduce the boundary effects of the plasma vessel, a third antenna configuration was used in setup C. Here, a double loop antenna was used with a radius smaller than that of the NL in order to investigate the significance of the NL position relative to the RF source. All experiments were conducted with an Argon plasma at a neutral gas pressure of typically $0.2 \mathrm{~Pa}$. The measurement plane is always that of the $\mathrm{NL}$, as indicated by the dashed line in figure 2 .

The choice of probe diagnostics has to be made carefully for the measurement of spatial plasma parameters near the NL. The plasma parameter regime changes from strongly magnetised far from the NL to an unmagnetized situation directly at the NL, including an intermediate regime of magnetized electrons and unmagnetized ions in between. Furthermore, the highly inhomogeneous magnetic field leads to a strong dependence of the field line connection length to the wall on the probe position. Together with rather low plasma densities, this can lead to the undesirable situation of Langmuir probes essentially behaving like double probes with the vessel wall as the second electrode. The saturation current to the probe then depends on the connection length as well as the plasma density, temperature and collection area. In past experiments, single and double Langmuir probes $[1,12]$ as well as Thomson scattering $[13,14]$ methods have been used for measurements of plasma density $n_{e}$ and electron temperature $T_{e}$. Further, phase resolved spectroscopy has been deployed to observe the electron dynamics [13]. In the present paper, a planar probe with a so called "guard ring" was predominantly used for saturation current measurements [15]. Due to a controlled expansion of the probe sheath, the electron collection area for this probe type is less susceptible to changes of the magnetic field than a cylindrical probe or a simple planar probe. For electron temperature and floating potential measurements, a double probe was utilized.

\section{Experimental Results}

\subsection{Setup A}

The observed radial plasma density profile for setup A (antenna far from NL) is depicted together with the absolute magnetic field in figures $3 \mathrm{a}$ and $3 \mathrm{~b}$. The position of the neutral loop $\left(R_{N L}\right)$ and of the helical antenna radius $\left(R_{A n t}\right)$ are marked with dashed lines. Compared to the radial profile in a homogeneous magnetic field, which is characterized by a density maximum slightly inwards of the antenna radius, a radial expansion of the plasma profile is observed, resulting in a peak density at $R_{A n t}<r<R_{N L}$. Further outwards, a density plateau at $r=120 \mathrm{~mm}$ is visible. These results are qualitatively consistent with those 

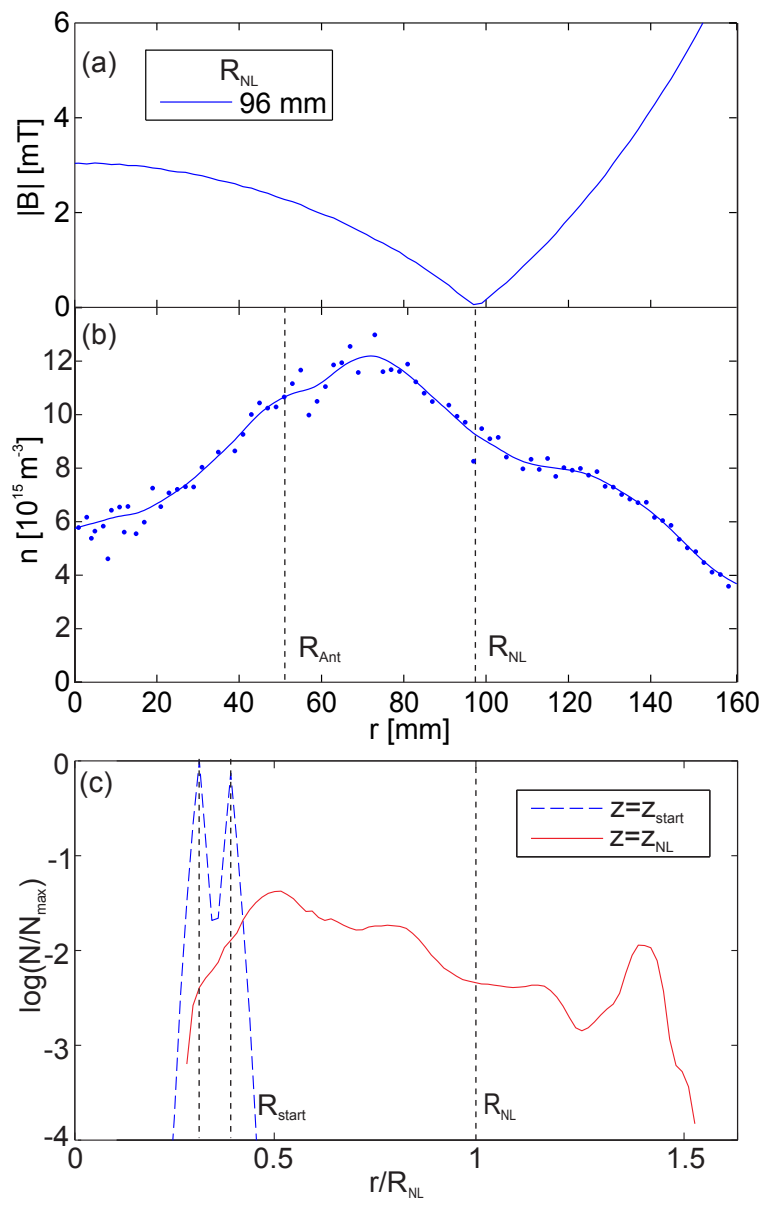

Fig. 3. Setup A: Absolute magnetic field strength (a) and plasma density in the NL plane, experiment (b) and simulation (c). The dashed lines denote the neutral loop and helicon antenna radius.

of numerical single-particle simulations which were conducted in the calculated magnetic field, shown in fig. 3c. In these simulations, the trajectories of electrons with starting points slightly inwards and outwards of the separatrix at the antenna location $\left(z=z_{\text {start }}\right.$, blue line) and a Maxwellian velocity distribution corresponding to the measured electron temperature of typically $2 \mathrm{eV}$ were calculated. Though the simulation includes neither electric fields, nor any other collective plasma effects, it still provides an overall picture of the effects which can be expected in the complex magnetic field. Specifically, particle trapping occurs in the mirror fields that surround the neutral point, which are responsible for a density maximum slightly inwards of the NL at $z=z_{N L}$ and a plateau slightly outwards. These findings indicate that a simple single-particle picture with neglected electric fields is sufficient to describe the situation at the NL when using an antenna far from the NL.

\subsection{Setup B}

This simple picture does not hold for the situation where the NL is within the skin depth $\delta_{\text {skin }}$ of the field supplied

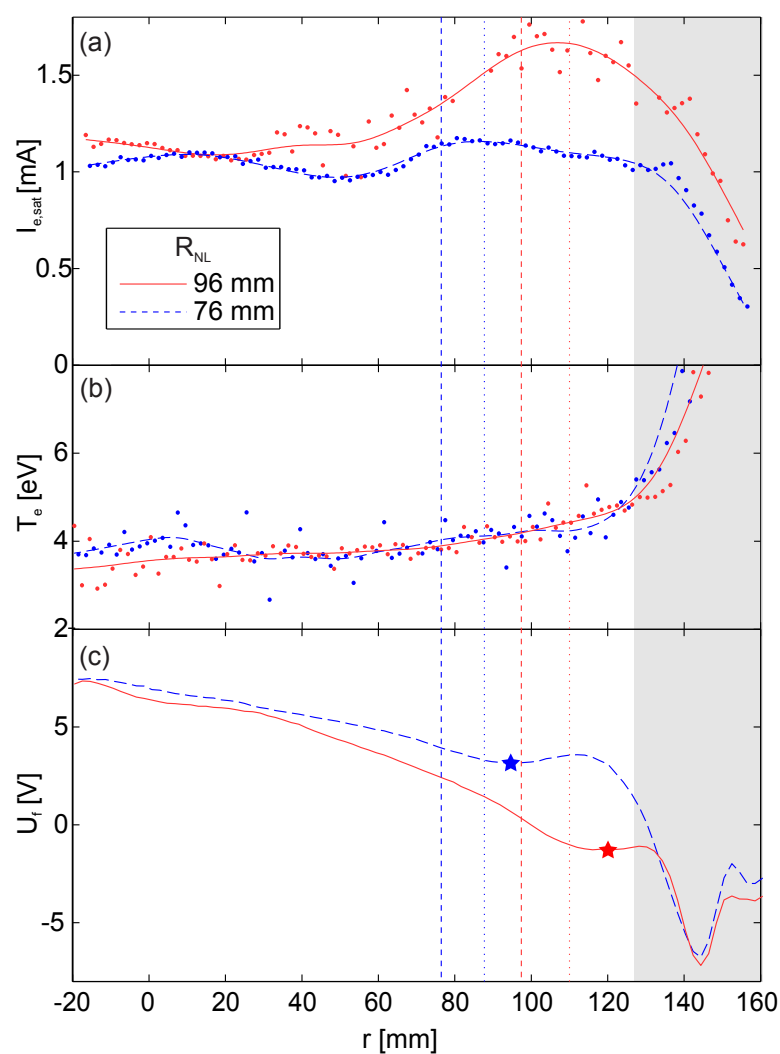

Fig. 4. Setup B: Electron saturation current and temperature (a,b) and floating potential (c) for two NL radii (dashed lines). The dotted lines indicate the density maxima, the gray area marks the capacitive sheath.

by rf antenna. Measurements using this setup are shown in fig. 4 for two different NL configurations. The antenna is located at $r=160 \mathrm{~mm}$, while the NL radius is $R_{N L}=76 \mathrm{~mm}$ (blue) and $96 \mathrm{~mm}$ (red), respectively. The ion saturation current profiles (a) exhibit clear maxima near, but slightly outwards of $R_{N L}$ with an amplitude that decreases exponentially with increasing distance of the NL from the antenna. This result deviates from results in previous experiments in which the maxima were always observed radially inside the position of the NL $[12,13]$. The exponential decay length $(\delta=15.5 \mathrm{~cm})$ corresponds to the skin depth at the measured densities $\left(n_{e} \approx 10^{15} \mathrm{~m}^{-3}, \delta_{\text {skin }}=17 \mathrm{~cm}\right)$, which indicates a connection between density peaks and the induced antenna electric field.

Further outwards (gray box), a sharp decline of the saturation current is observed near the antenna, which is attributed to the presence of a capacitive sheath near the antenna. In this region, the probe characteristic is distorted by the rf, which leads to unreliable evaluation of the probe characteristics, especially with respect to the (overestimated) electron temperature.

In contrast to previous experiments, the measured temperature profiles (b) are essentially flat outside of the antenna sheath. Okraku-Yirenkyi et al. observed that the electron temperature almost doubles in a narrow area around the NL, which supports the hypothesis of increased lo- 


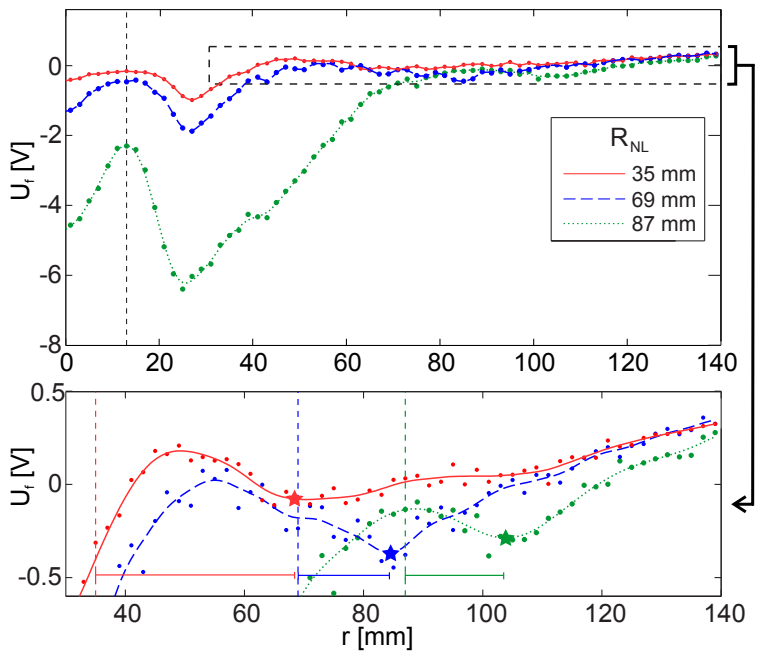

Fig. 5. Setup C: Floating potential profile (top) and zoomed region (dashed box, bottom) for three NL radii. The dotted black line denotes the antenna position, the colored lines correspond to the NL positions.

cal plasma production due to anomalous resistivity [12]. The absence of these maxima in the recorded profiles suggests that another heating process may be of importance. This assumption is further supported by the measured floating potential profiles (c). In addition to the large potential minimum in the direct vicinity of the antenna at $r=145 \mathrm{~mm}$, the profiles exhibit local minima at the density peaks (stars). These potential dips may indicate a fast electron population which can be attributed to an enhanced induced ring current. The radial positions of these points coincide with those of the electron cyclotron resonance $(B=0.48 \mathrm{mT}$ for $f=13.56 \mathrm{MHz})$, thus hinting at electron cyclotron resonance (ECR) heating. This, however, is not the case for the modified antenna setup C, therefore rendering ECR heating an unlikely candidate for the NLD generation.

\subsection{Setup C}

The measurements performed for setup $\mathrm{C}$ with an antenna radius smaller than that of the NL are shown in fig. 5. At a similar rf power to setup B $(60 \mathrm{~W})$, peak plasma densities of $10^{17} \mathrm{~m}^{-3}$ were recorded which correspond to a skin depth of $\delta_{\text {skin }}=1.7 \mathrm{~cm}$. Since the NL is farther from the antenna than this distance, the increase in density near the NL (not shown) is minimal. Nevertheless, a similar floating potential structure as in setup B can be observed in fig. 5. As in the previous case, distinct potential minima are observed close to the antenna at a fixed position. Additionally, local minima can be seen (zoomed area, stars) which correlate with the NL position and provide evidence of a local fast electron population.

\section{Summary and Conclusion}

The plasma dynamics at the NL is observed to be very much determined by its distance to the rf antenna. Measurements with an antenna far from the magnetic null (setup A) exhibit density maxima radially inwards of the NL position. These results are consistent with a simple single particle simulation in a static magnetic field and are associated with particle trapping in the mirror fields which surround the neutral point. When the rf antenna is located near the NL (setup B), density maxima are observed at a radial location slightly outside the magnetic null; they correlate with the amplitude of the induced rf field. The observed temperature profiles do not support the hypothesis of a thermal heating mechanism at the NL. The temperature profiles are essentially flat in the vicinity of the NL and cannot account for the high densities measured. Instead, the observed floating potential dips indicate the local presence of non-thermal electrons. This supports the picture of a locally enhanced plasma current at the neutral point due to the vanishing magnetic field perpendicular to the induced electric field.

\section{References}

1. H. Tsuboi, Jap. J. Appl. Phys. 34(5A), 2476 (1995), ISSN 0021-4922

2. T. Gans, D.L. Crintea, D. O'Connell, U. Czarnetzki, J. Phys. D: Appl. Phys. 40(615), 4508 (2007)

3. Z. Yoshida, Jap. J. Appl. Phys. 34(8A), 4213 (1995), ISSN 0021-4922

4. W. Chen, Y. Morikawa, M. Itoh, T. Hayashi, K. Sugita, H. Shindo, T. Uchida, J. Vac. Sci. Technol. A 17(5), 2546 (1999), ISSN 0734-2101

5. H. Asakura, K. Takemura, Z. Yoshida, T. Uchida, Jap. J. Appl. Phys. 36(7A), 4493 (1997)

6. M. Vural, R.P. Brinkmann, J. Phys. D: Appl. Phys. 40(2), 510 (2007), ISSN 0022-3727

7. Z. Yoshida, H. Asakura, H. Kakuno, J. Morikawa, K. Takemura, S. Takizawa, T. Uchida, Phys. Rev. Lett. 81(12), 2458 (1998)

8. T. Uchida, J. Phys. D: Appl. Phys. 41(8), 083001 (2008), ISSN 0022-3727

9. Y. Celik, D.L. Crintea, D. Luggenholscher, U. Czarnetzki, T. Ishijima, H. Sugai, Phys. Plasmas 18(2), 022107 (2011)

10. C.M. Franck, O. Grulke, T. Klinger, Phys. Plasmas 9(8), $3254(2002)$

11. C.M. Franck, O. Grulke, T. Klinger, Phys. Plasmas 10(1), $323(2003)$

12. Y. Okraku-Yirenkyi, Y.M. Sung, M. Otsubo, C. Honda, T. Sakoda, J. Vac. Sci. Technol. A 19(5), 2590 (2001)

13. D. O'Connell, T. Gans, D.L. Crintea, U. Czarnetzki, N. Sadeghi, Plasma Sources Sci. Technol. 17, 024022 (12 pp.) (2008)

14. Y.M. Sung, T. Sakoda, J. Vac. Sci. Technol. A 20(6), 1964 (2002)

15. N.S. Braithwaite, J.P. Booth, G. Cunge, Plasma Sources Sci. Technol. 5(4), 677 (1996) 